\title{
CONSIDERACIONES MORFOLÓGICAS Y TAXONÓMICAS SOBRE ISCHNOCHITON (ISCHNOCHITON) RISSOI (PAYRAUDEAU, 1826) (MOLLUSCA, POLYPLACOPHORA)
}

\author{
P. Carmona Zalvide (*) y F. J. García García (**)
}

\begin{abstract}
RESUMEN
La amplia variabilidad de ornamentación de Ischnochiton (Ischnochiton) rissoi (Payraudeau, 1826) ha determinado que ejemplares de esta especie se hayan descrito como especies diferentes. En este artículo, además de revisar y discutir las diversas opiniones existentes, se ha realizado un estudio con microscopía electrónica de barrido de numerosos ejemplares y se ha observado la gran heterogeneidad de $I$. (I.) rissoi en relación al tipo de ornamentación de la concha y del perinoto, aunque se han podido determinar ciertos caracteres comunes que permiten identificarlos como pertenecientes a una única especie.
\end{abstract}

Palabras clave: Ischnochiton rissoi, Polyplacophora, Mollusca, Taxonomía, Península Ibérica.

\section{ABSTRACT \\ Morphological and taxonomic remarks on Ischnochiton (Ischnochiton) rissoi (Payraudeau, 1826) (Mollusca, Polyplacophora)}

The morphological variability of Ischnochiton (Ischnochiton) rissoi (Payraudeau, 1826) has produced taxonomic discrepancies. In this paper, besides a taxonomic revision, the morphology of valves, perinotum and radula of several specimens of I.(I.) rissoi is studied using Scanning Electron Microscopy. Although a notable morphological variability is present in this species, however, some common features identify all the varieties as an only taxon.

Key words: Ischnochiton rissoi, Polyplacophora, Mollusca, Taxonomy, Iberian Peninsula.

\section{Introducción}

Ischnochiton (Ischnochiton) rissoi (Payraudeau, 1826) ha ocasionado contrariedades taxonómicas a causa de la variabilidad que presenta la ornamentación del tegmento. Reeve (1847) describe Chiton mediterraneus, aunque mantiene como especie válida a Chiton rissoi, y los diferencia por las estrí- as lisas o granulosas de las valvas terminales. Capellini (1859), que también acepta I. (I.) rissoi, describe una especie nueva, Chiton meneghinii, distinguiéndola de I. (I.) rissoi por presentar la superficie finamente dentada. Monterosato (1878) únicamente considera como especie valida $C$. mediterraneus y sinonimiza $C$. rissoi y $C$. meneghinii clasificándolas como variedades, y, a su vez, des-

* Laboratorio de Zoología Marina, Departamento de Bioloxía Animal, Facultade de Bioloxía, Univ. Santiago de Compostela, 15706 Santiago de Compostela (Spain). E-mail: bavituco@usc.es

** Departamento de Fisiología y Biología Animal, Facultad de Biología, Univ. Sevilla, Apdo. 1095, 41080 Sevilla. (Spain). Email: fjgarcia@cica.es 
cribe la variedad fragilis, caracterizándola por presentar una escultura menos marcada. Pilsbry (1892) admite como especie a $I$. (I.) rissoi y las variedades meneghinii, por poseer el tegumento fuertemente esculpido, y fragilis por presentarlo menos apreciable, mientras que considera $C$. mediterraneus sinónimo de I. (I.) rissoi. Ancey (1898), que admite Leptochiton meneghinii, propone una nueva variedad para esta especie: dautzenbergi.

Mediante microscopía electrónica de barrido se ha realizado un estudio comparativo de diferentes ejemplares de $I$. (I.) rissoi con características ornamentales del tegmento muy distintas, que varían desde estrías onduladas a rectas y de poco marcadas a patentes, en las áreas centrales de las placas intermedias, y desde líneas concéntricas a radiales, tanto en la placa cefálica como en el área postmucral de la placa caudal.

\section{Material y métodos}

Los ejemplares estudiados han sido recolectados en el litoral atlántico de la Península Ibérica (Cádiz-Gibraltar), Mallorca (islas Baleares) y Tetuán (Marruecos) (fig. 1). Se han capturado de forma directa en las zonas mediolitoral e infralitoral, en ésta con equipos de inmersión autónoma. A su vez se han estudiado los ejemplares de la colec- ción del Museo Nacional de Ciencias Naturales de Madrid (MNCN).

Para el estudio de las partes duras se introduje-

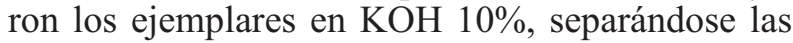
valvas, escamas y espículas del perinoto y la rádula. La estructura de las diferentes partes se observó mediante el uso de microscopía electrónica de barrido (Philips XL-20).

\section{Resultados}

Clase POLYPLACOPHORA Gray, 1821

Orden NEOLORICATA Bergenhayn, 1955

Suborden ISCHNOCHITONINA Bergenhayn, 1930

Familia ISCHNOCHITONIDAE Dall, 1889

Ischnochiton (Ischnochiton) rissoi (Payraudeau, 1826)

SinONIMIAS:

Chiton mediterraneus (Gray MS) Reeve, 1847

Chiton meneghinii Capellini, 1859

Chiton carinatus Issel, 1870

Chiton dautzenbergi Ancey, 1898

Chiton fragilis Monterosato, 1878

MATERIAL ESTUDIADO: El total de ejemplares estudiados ha sido de 210. Se especifica la localidad, número de ejemplares, el tamaño máximo de los ejemplares recolectados, fecha y profundidad.
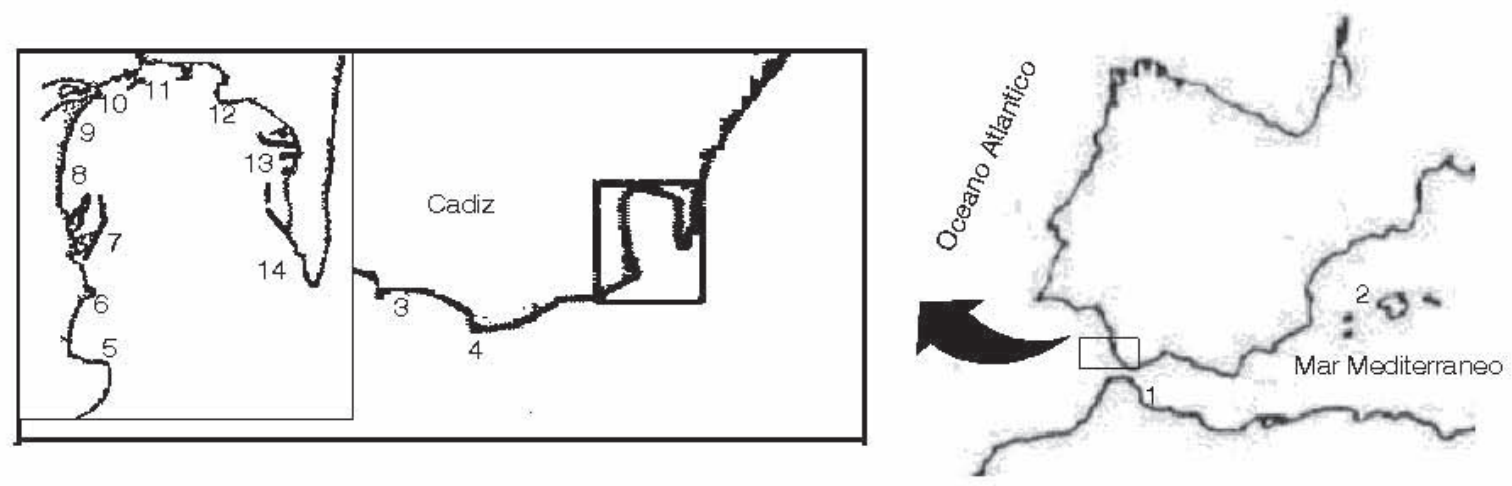

Fig 1.-Estaciones de muestreo: 1. Tetuán (Marruecos); 2. Mallorca (Islas Baleares); 3. Playa del Chorro (Cádiz); 4. Isla de Tarifa (Cádiz); 5. Isla de las Palomas (Cádiz); 6. La Ballenera (Cádiz); 7. Punta de San García (Cádiz); 8. Ensenada de Cucareo (Cádiz); 9. Saladillo (Cádiz); 10. Puerto de Algeciras (Cádiz); 11. Punta de Paredones (Cádiz); 12. Punta del Gallo del Mirador (Cádiz); 13. San Felipe (Cádiz); 14. Punta Europa (Gibraltar).

Fig 1.- Sampling stations. 1. Tetuán (Marruecos); 2. Mallorca (Islas Baleares); 3. Playa del Chorro (Cádiz); 4. Isla de Tarifa (Cádiz); 5. Isla de las Palomas (Cádiz); 6. La Ballenera (Cádiz); 7. Punta de San García (Cádiz); 8. Ensenada de Cucareo (Cádiz); 9. Saladillo (Cádiz); 10. Puerto de Algeciras (Cádiz); 11. Punta de Paredones (Cádiz); 12. Punta del Gallo del Mirador (Cádiz); 13. San Felipe (Cádiz); 14. Punta Europa (Gibraltar). 
Playa del Chorro, Cádiz: 6 ej., 18 x 9 mm, 07/92 (intermareal). Isla de Tarifa, Cádiz: 1 ej., $3 \times 1.5 \mathrm{~mm}, 07 / 90$ (7 m); 6 ej., 15 x 6 mm, 03/91 (10 m); 1 ej., $18 \times 7$ mm, 04/92 (3 m); 6 ej., $15.1 \times 8 \mathrm{~mm}, 01 / 93(9 \mathrm{~m})$. Isla de las Palomas, Cádiz: 4 ej., 13 x $6 \mathrm{~mm}, 08 / 91(8 \mathrm{~m}) ; 9$ ej., 13 x $6 \mathrm{~mm}, 09 / 92(12 \mathrm{~m}) ; 1$ ej., 10 x $5 \mathrm{~mm}, 11 / 93$ (9 m). La Ballenera, Cádiz: 10 ej., 12 x $6 \mathrm{~mm}$, 04/92 (5-9 m). Punta de S. García, Cádiz: 1 ej., 5 x 3 mm, 07/91 (4-5 m); 14 ej., 14 x 8 mm, 11/91 (5 m); 1 ej., 11.5 x 5 mm, 09/92 (12 m); 6 ej., 12 x 6 mm, 09/92 (12 m). Ensenada de Cucareo, Cádiz: 3 ej., 17 x 7 mm, 09/92 (3 m); 3 ej., 12.5 x 5 mm, 09/92 (5 m); 81 ej., 17 x 8.5 mm, 01/93 (5 m); 1 ej., 7.5 x $4 \mathrm{~mm}, 09 / 93$ (3 m). Saladillo, Cádiz: 1 ej., 13 x $6.5 \mathrm{~mm}, 07 / 93$ (5 m). Puerto de Algeciras, Cádiz: 1 ej., 13 x 6 mm, 07/91 (5 m); 1 ej., 9 × 6 mm, 07/91 (3 m); 2 ej., 8 × 3 mm, 08/91 (6 m); 1 ej., 13 x $6.5 \mathrm{~mm}, 07 / 93$ (5 m). Punta de Paredones, Cádiz: 1 ej., 3.5 x $2.5 \mathrm{~mm}, 07 / 90$ (14 m); 7 ej., $11 \times 5 \mathrm{~mm}, 07 / 91$ (9 m); 1 ej., 9 x 4.5 mm, 07/91 (16 m). Punta del Gallo del Mirador, Cádiz: 1 ej., 10 x 4.5 mm, 08/92 (5 m). San Felipe, Cádiz: 2 ej., 7 x 3 mm, 07/91 (5 m). Punta Europa, Gibraltar: 1 ej., 3.5 x 2 mm, 04/92 (25 m); 1 ej., 14.5 x 6.5 mm, 07/93 (15 m). Tetuán (Marruecos): 14 ej., 12.5 x $6.4 \mathrm{~mm}, 05 / 93$ (5 m). MNCN 1503/130 (Mahón, Menorca, Baleares): 1 ej., 12 × $7 \mathrm{~mm}$; MNCN 1503/138 (Cabrera, Baleares): 2 ej., $14 \times 6.5 \mathrm{~mm}$ MNCN 1503/365 (Cádiz): 6 ej., 17.5 x 8 mm; MNCN 1503/366 (Palma, Baleares): 1 ej., 17 x 8.5 mm; MNCN 1503/374 (Cádiz): 2 ej., 18 x 8.5 mm; MNCN 1503/376 (isla de Aire): 1 ej., 13 x 6 mm; MNCN 1503/377 (España): 2 ej., 25.5 x 12 mm;
MNCN 1503/378 (Cabrera, Baleares): 4 ej., 10 x 6 mm; MNCN 1503/423 (Santander): 3 ej., $9 \times 12 \mathrm{~mm}$.

DESCRIPCIÓN: Se han recolectado ejemplares de tamaños comprendidos entre $3.0 \times 1.5 \mathrm{~mm}$ y $18 \times 9$ $\mathrm{mm}$. El aspecto del cuerpo es oblongo, ligeramente carenado, regularmente arqueado, con conchas consistentes, que exhiben una gran variabilidad en la ornamentación del tegmento, en especial en las tonalidades, ya que la mayoría de los ejemplares presentan combinación de colores. El perinoto es relativamente ancho, constituido por escamas imbricadas con apariencia de piel de serpiente y con un fleco marginal patente.

La placa oral muestra el borde anterior de forma semicircular y el posterior triangular, con un ángulo obtuso muy marcado. Las valvas intermedias presentan una forma rectangular, excepto la segunda que es trapezoidal. Las áreas laterales destacan principalmente por el cambio de ornamentación del tegmento, ya que se encuentran ligeramente sobre el área central. La valva caudal es semicircular; el mucro es bastante prominente y se localiza en la zona central de la valva. Presenta una fuerte pendiente cóncava.

Tabla 1. - Variabilidad morfológica de Ischnochiton (Ischnochiton) rissoi.

Table 1. - Morphological variability of Ischnochiton (Ischnochiton) rissoi

\begin{tabular}{lcccc}
\hline & Forma 1 & Forma 2 & Forma 3 & Forma 4 \\
\hline Localidad & MNCN & Mallorca & Algeciras & $\begin{array}{c}\text { Tetuán } \\
\text { Gibraltar }\end{array}$ \\
& & & Gibraltar & MNCN \\
& & & $18 \times 9$ & $12.5 \times 6.4$ \\
Tamaño máximo $(\mathrm{mm})$ & $18 \times 9$ & $14 \times 7.5$ & variable & variable \\
Color & blanco & verdoso & 5.5 & 6.6 \\
Diámetro medio esteta $(\mu \mathrm{m})$ & 5.06 & 6.16 & $\sigma: 0.91$ & $\sigma: 0.25$ \\
& $\sigma: 0.21$ & $\sigma: 0.81$ & 8.1 & 13.33 \\
Distancia media entre estetas de filas & 7.55 & 8.26 & $\sigma: 1.78$ & $\sigma: 4.3$ \\
paralelas $(\mu$ m) & $\sigma: 1.58$ & $\sigma: 2.53$ & 16.19 & 18.3 \\
Distancia media entre estetas de una & 11.5 & 20.4 & $\sigma: 3.05$ & $\sigma: 5.36$ \\
misma fila $(\mu$ m) & $\sigma: 2.0$ & $\sigma: 4.68$ & $10-13 / 1 / 9-12$ & $8-11 / 1 / 8-9$ \\
Fórmula línea de inserción & $10 / 1 / 11$ & $11 / 1 / 10$ & 133.6 & 131.03 \\
Tamaño medio de escamas dorsales $(\mu \mathrm{m})$ & 160 & 162.5 & $\sigma: 8.35$ & $\sigma: 13.2$ \\
& $\sigma: 12.8$ & $\sigma: 8.25$ & 58.2 & 56.64 \\
Tamaño medio espículas ventrales $(\mu \mathrm{m})$ & 60.2 & 47.3 & $\sigma: 5.9$ & $\sigma: 3.08$ \\
& $\sigma: 4.1$ & $\sigma: 3.6$ & $15-24$ & $17-20$ \\
Rango del $n^{\circ}$ de costillas en escamas dorsales & $9-11$ & $17-20$ & $17-21$ & $22-24$ \\
Rango del $n^{\circ}$ de branquias & $($ seco) & $30-31$ & 58.6 & 60 \\
Tamaño medio diente raquídeo $(\mathrm{mm})$ & rádula ausente & 62.5 & 75.4 & 82 \\
Tamaño medio diente primer lateral $(\mathrm{mm})$ & rádula ausente & 85.88 & \\
\hline
\end{tabular}




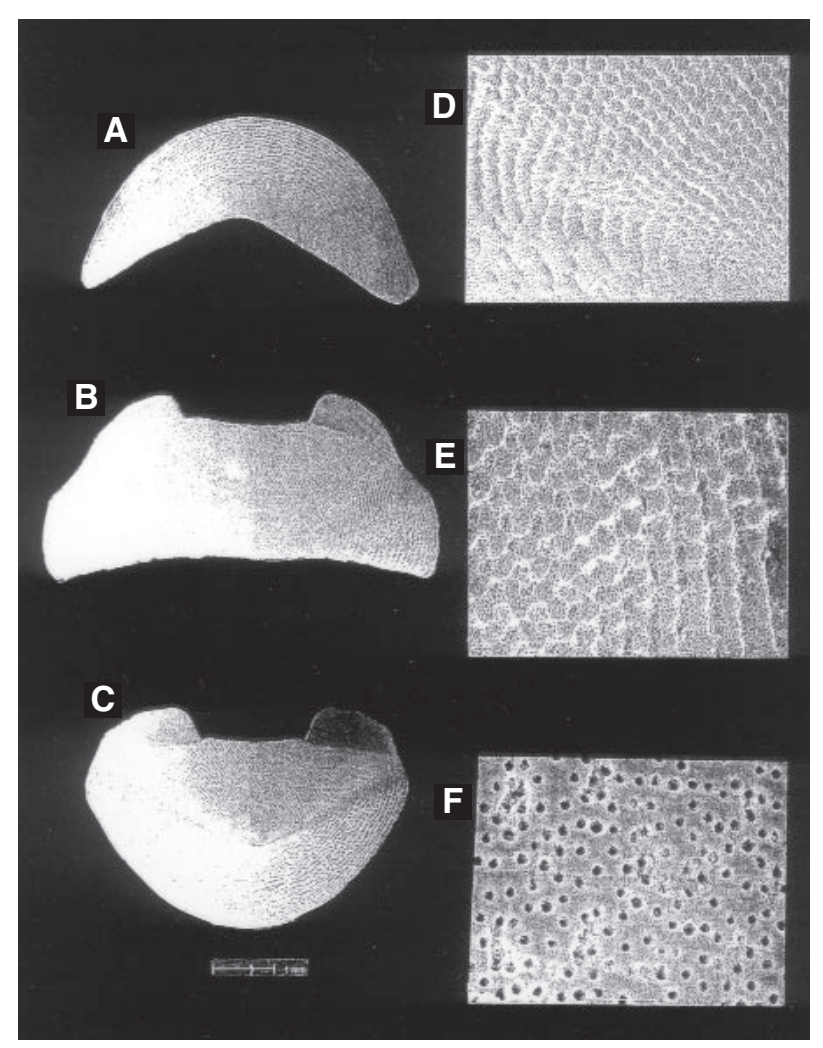

Fig. 2.- Ischnochiton (I.) rissoi (Museo Nacional de Ciencias Naturales de Madrid, forma 1). A) Valva I; B) valva IV; C) valva VIII; D) disposición de los tubérculos en las distintas zonas de la valva IV; E) disposición de las estetas en la zona pleural; F) disposición de las estetas en la zona jugal.

Fig. 2.- Ischnochiton (I.) rissoi (Museo Nacional de Ciencias Naturales de Madrid, forma 1). A) Valve I; B) valve IV; C) valve VIII; D) location of tubercles on the valve IV; E) location of aesthetes on the pleural area; F) location of aesthetes on the jugal area.

La ornamentación del tegmento es muy variada, observándose un abanico gradual en la estructura, de forma que puede presentarse desde muy débilmente marcada por no ofrecer granulación, hasta fuertemente trazada por presentar de forma patente esta granulación. Se van a definir cuatro formas puntuales de la gama de estructuras posibles (Tabla 1).

La ornamentación es similar en la placa cefálica, las áreas laterales de las valvas intermedias y la zona postmucral.

Forma 1: Los gránulos se encuentran bien definidos y tienden a formar cadenas concéntricas semicirculares desde el ápice o mucro de las placas (fig. 2).

Forma 2: Los gránulos forman cordones, de manera que pierden la identidad como tales gránu-

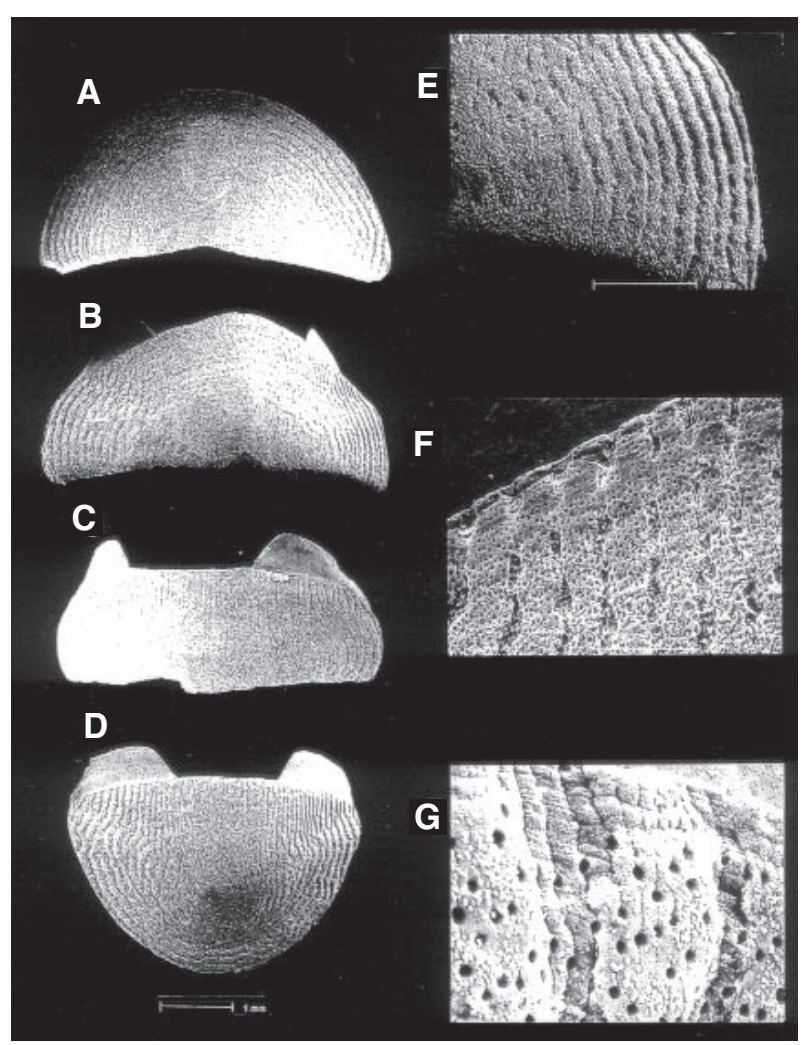

Fig. 3.-Ischnochiton (I.) rissoi (Mallorca, forma 2). A) Valva I; B) valva II; C) valva IV; D) valva VIII; E) disposición de los tubérculos en el área lateral; F) disposición de los tubérculos en la zona pleural; G) disposición de las estetas.

Fig. 3.- Ischnochiton (I.) rissoi (Mallorca, forma 2). A) Valve I; B) valve II; C) valve IV; D) valve VIII; E) location of tubercles on lateral area; F) location of tubercles on pleural area; G) location of aesthetes.

los, su disposición es de igual forma que los anteriores, semicircular con ordenación concéntrica y distribuidas desde el ápice o mucro (fig. 3).

Forma 3: De estructura igual que la anterior, pero con los cordones distribuidos sólo en la zona terminal de las placas (fig. 4).

Forma 4: Gránulos muy marcados que, a pesar de formar las cadenas concéntricas, destacan más las cadenas longitudinales, dispuestas radialmente desde el ápice o mucro hasta la zona terminal (fig. 5).

El área central de las placas intermedias y la zona anteromucral ofrecen una ornamentación similar.

Forma 1: La zona jugal es lisa y los gránulos aparecen en la zona pleural, éstos forman cadenas 


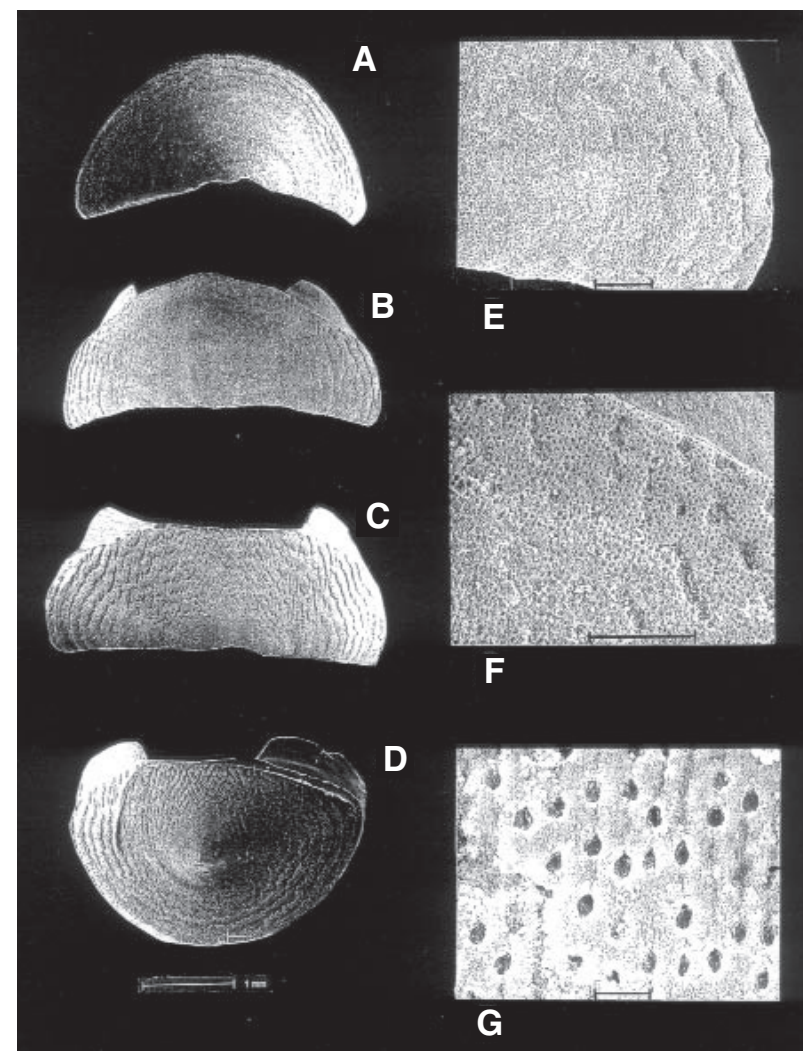

Fig. 4.- Ischnochiton (I.) rissoi (Algeciras, forma 3). A) Valva I; B) valva II; C) valva IV; D) valva VIII; E) disposición de los tubérculos en el área lateral; F) disposición de los tubérculos en la zona pleural; G) disposición de las estetas.

Fig. 4.- Ischnochiton (I.) rissoi (Algeciras, forma 3). A) Valve I; B) valve II; C) valve IV; D) valve VIII; E) location of the tubercles on lateral area; F) location of tubercles on the pleural area; G) location of aesthetes.

convergentes hacia la zona anterior de la placa (fig. 2B).

Forma 2: Los cordones tienden a divergir en la zona jugal, sin embargo en la pleural comienzan a converger en su inicio para continuar rectos hacia la zona terminal (figs. 3B y $3 \mathrm{C}$ ).

Forma 3: Los gránulos de la zona jugal están poco marcados y tienden a unirse en una estructura encrespada. En la pleural los cordones son patentes en el área próxima a los bordes; se inician a nivel del área lateral de forma convergente y continúan rectos a medida que se aproximan a la zona anterior, donde se observan con estructura encrespada (figs. 4B y 4C).

Forma 4: Se distinguen cordones bien marcados, ordenados longitudinalmente de forma parale-

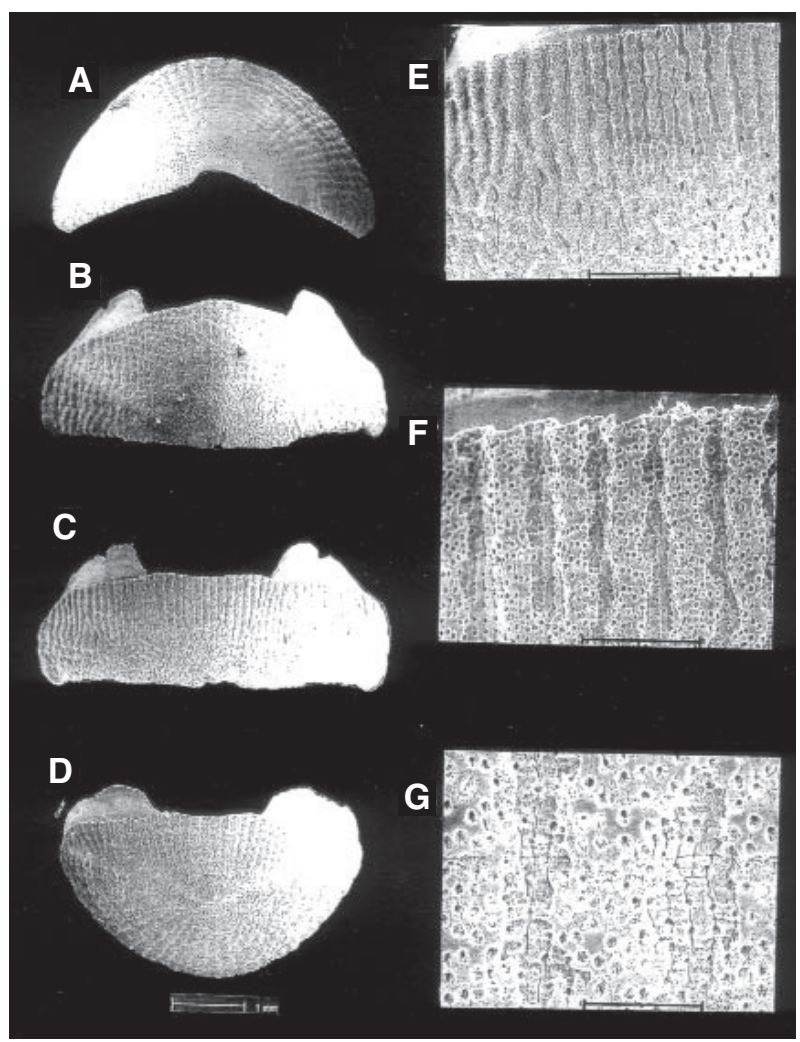

Fig. 5.- Ischnochiton (I.) rissoi (Gibraltar, forma 4). A) Valva I; B) valva II; C) valva IV; D) valva VIII; E) disposición de los tubérculos en las áreas central y lateral; F) disposición de los tubérculos en la zona pleural; G) disposición de las estetas.

Fig. 5.-Ischnochiton (I.) rissoi (Gibraltar, forma 4). A) Valve I; B) valve II; C) valve IV; D) valve VIII; E) location of the tubercles on the central and lateral areas; F) location of tubercles on the pleural area; G) location of the aesthetes.

la en la zona jugal. En la pleural, se disponen de igual manera, variando el inicio, a nivel de la intersección con las áreas laterales, en el cual convergen levemente (figs. 5B y 5C).

Las estetas se disponen en líneas. Se encuentran separadas en una misma fila entre $11.5 \mu \mathrm{m}$ ( $\sigma: 1.57)$ y $18.3 \mu \mathrm{m}$ (б: 5.36) en diferentes ejemplares. La distancia entre líneas oscila sobre $7.55 \mu \mathrm{m}(\sigma: 1.58)$ y $13.33 \mu \mathrm{m}$ ( $\sigma: 4.3$ ) (Tabla 1). El diámetro de las estetas varía mínimamente en las diferentes formas, Forma $1=5.06 \mu \mathrm{m}(\sigma: 0.21)$ (fig. $2 F)$, Forma $2=6.16$ $\mu \mathrm{m}(\sigma: 0.81)$ (fig. 3G), Forma $3=5.5 \mu \mathrm{m}(\sigma: 0.91)$ (fig. 4G) y Forma $4=6.6 \mu \mathrm{m}(\sigma: 0.25)$ (fig. $5 \mathrm{G}$ ).

Articulamento. La coloración es blanca en la zona jugal y tiende a variar el tono hacia las líneas 


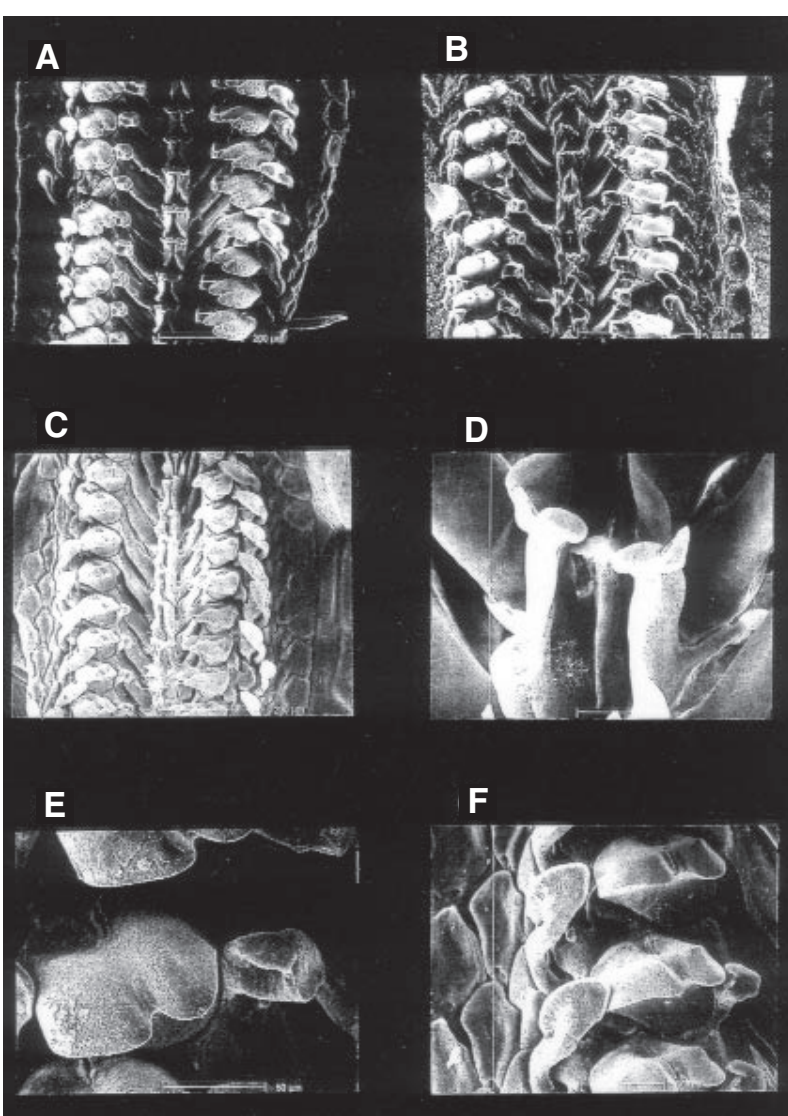

Fig. 6.- Rádula de Ischnochiton (I.) rissoi: A) ejemplar de Mallorca; B) ejemplar de Gibraltar; C) ejemplar de Algeciras; D) dientes raquídeo y primer lateral; E) placa uncinada del diente mayor lateral y apófisis de la base de la placa; F) dientes lateral mayor, plumoso y los externos.

Fig. 6. - Radula of Ischnochiton (I.) rissoi: A) specimen from Mallorca; B) specimen from Gibraltar; C) specimen from Algeciras; D) rachidian and first lateral tooth; E) uncinal plate and appendix of its base; F) major lateral tooth, spatulated and marginal teeth.

de inserción en función de la coloración del tegmento. Los aleros son sólidos y los dientes se encuentran ligeramente ondulados. La forma de las apófisis varían en las distintas valvas: triangulares en II, III y IV; trapezoidal en V, VI y VII; y rectangular en VIII. La fórmula de las líneas de inserción es: $10-13 / 1 / 9-12$ (Tabla 1 ).

Cintura. El perinoto muestra una coloración variada en los distintos ejemplares. Está constituido por escamas imbricadas de aspecto oval, ornamentadas con costillas. En un mismo individuo varían de tamaño desde el borde anterior al marginal, la

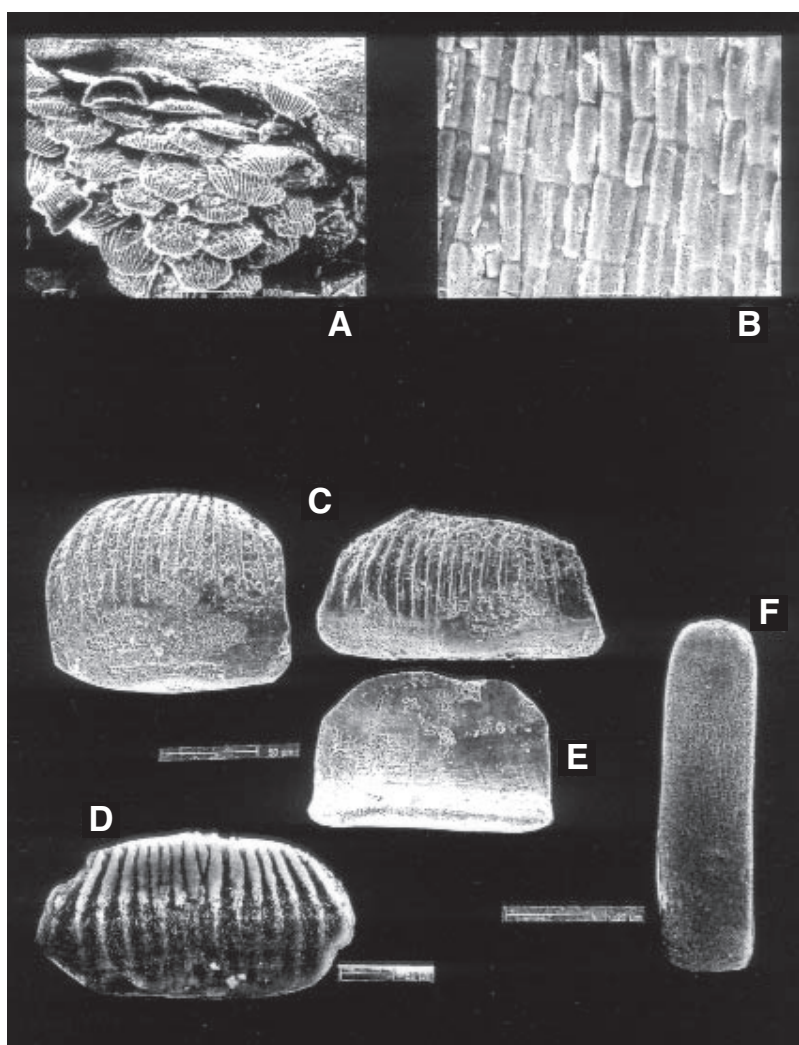

Fig. 7.- Ischnochiton (I.) rissoi (Algeciras). A) Disposición de las escamas dorsales; B) disposición de las escamas ventrales; C-D) escamas dorsales, vista dorsal; E) escama dorsal, vista ventral; F) escama ventral.

Fig. 7.- Ischnochiton (I.) rissoi (Algeciras). A) Location of dorsal scales; B) location of ventral scales; C-D) dorsal view of dorsal scales; E) ventral view of dorsal scales; F) ventral scales.

media oscila entre $133.6 \mu \mathrm{m}(\sigma: 8.35)$ y $162,5 \mu \mathrm{m}$ ( $\sigma: 8.29)$. Las costillas pueden ser finas o gruesas; su número ha variado según las distintas formas definidas: Forma $1=9-11$ (fig. 8A); Forma $2=17$ 20 (figs. 8B, 8I-K); Forma 3= 15-24 (fig. 7); $\mathrm{y}$ Forma $4=17-20$ (fig. 7C). Entre las escamas pueden presentar espículas de forma cilíndricas con un pedúnculo largo bastante más estrecho. En la zona marginal aparecen dos tipos de espículas de diferentes formas y tamaños. Las más abundante ofrecen una figura ligeramente cónica al presentar el extremo terminal apuntado y se encuentran ornamentadas con estriaciones longitudinales, su tamaño varía entre $55 \times 20 \mu \mathrm{m}$ y 78 × $23 \mu \mathrm{m}$; y las menos abundantes son lisas y alargadas, su tamaño 


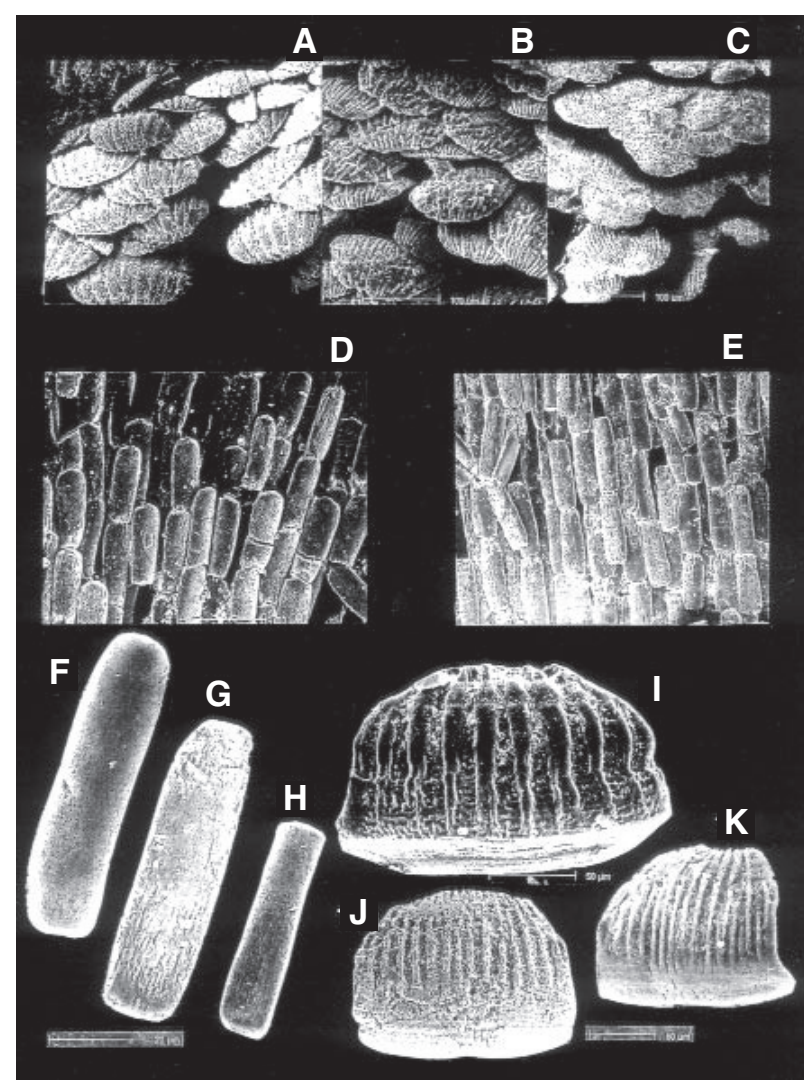

Fig. 8.- Disposición de las escamas dorsales y de I. (I.) rissoi: A) ejemplar del Museo Nacional de Ciencias Naturales de Madrid; B) ejemplar de Mallorca; C) ejemplar de Gibraltar. Disposición de las escamas ventrales de I. (I.) rissoi: D) ejemplar de Mallorca; E) ejemplar de Gibraltar. Escamas ventrales de I. (I.) rissoi: F-G) ejemplar del Museo Nacional de Ciencias Naturales de Madrid; H) ejemplar de Gibraltar; I-K) ejemplar de Mallorca.

Fig. 8. - Location of dorsal scales of $I$. (I.) rissoi: A) specimen from Museo Nacional de Ciencias Naturales de Madrid; B) specimen from Mallorca; C) specimen from Gibraltar. Location of ventral scales of $I$. (I.) rissoi: D) specimen from Mallorca; E) specimen from Gibraltar. Ventral scales of $I$. (I.) rissoi: F-G) specimen from Museo Nacional de Ciencias Naturales de Madrid; H) specimen of Gibraltar; I-K) specimen of Mallorca.

oscila entre 110 x $10 \mu \mathrm{m}$ y 138 × $12 \mu \mathrm{m}$. Las escamas ventrales, de color blanco, son más pequeñas que las dorsales; generalmente presentan una forma rectangular, con los borde terminales redondeados, su longitud varía entre 45 y $80 \mu \mathrm{m}$ (Tabla 1) (figs. 7F, 8D-G).

Branquias. Son del tipo holobranquial abanal, se disponen desde el nivel de la valva II hasta las inmediaciones de la inserción entre las placas VII-VIII.

Rádula (fig. 6). El diente raquídeo es alargado y estrecho, con una altura media de $60.3 \mu \mathrm{m}$ ( $\sigma$ : 1.52). El primer diente lateral es más ancho y alto, con una altura media de $81.1 \mu \mathrm{m}$ ( $\sigma: 4.3)$; se caracteriza por exhibir una protuberancia de forma triangular en la zona terminal y exterior del diente. El diente mayor lateral muestra una placa uncinada bicúspide con una pequeña apófisis cilíndrica en la base de ésta. El diente plumoso es de un tamaño bastante más pequeño que la placa uncinada del diente mayor lateral, se sitúa siempre por debajo ésta.

Biología: Se han encontrado generalmente en piedras sueltas de zonas rocosas y en playas arenosas. Todas las piedras donde se han colectado se caracterizaban por estar cubiertas de algas calcáreas. Los individuos se disponían en la zona inferior protegiéndose de la luz. En un mismo hábitat se han encontrado ejemplares de las diferentes formas descritas. Los ejemplares recolectados han presentado una distribución batimétrica desde la zona mediolitoral hasta $25 \mathrm{~m}$ de profundidad.

\section{Discusión}

Chiton rissoi fue descrito por Payraudeau (1826). Reeve (1847) aceptó esta especie y citó como nueva a $C$. mediterraneus, que diferenció de la anterior por la coloración y la ornamentación, ondulada y de forma rugosa, del tegmento de las valvas terminales y de las placas intermedias. La descripción la realizó a partir de ejemplares de la colección de Gray, sin embargo este autor no la citó en ninguno de sus trabajos posteriores.

Shuttleworth (1853) admitió las dos especies, las diferenció por la ornamentación que presenta el tegmento y las clasificó en géneros diferentes: Ischnochiton rissoi y Leptochiton mediterraneus.

Capellini (1859) únicamente consideró a C. rissoi y describió la especie C. meneghinii, que distinguió por presentar las áreas bastantes definidas, la superficie finamente dentada, con las valvas extremas y áreas laterales radiadas y rizadas concéntricamente, y 10/1/12 como fórmula de líneas de inserción.

Monterosato (1878) no consideró justificables las diferencias entre C. rissoi y C. meneghinii para distinguir dos especies por lo que consideró a $C$. meneghinii como una variedad de la anterior con una ornamentación más marcada. Además añadió 
que en realidad ésta es la que se correspondería con la descripción efectuada por Payraudeau (1826), quien también mencionó la gran variedad en la ornamentación del tegmento de $C$. rissoi. Por otra parte, propuso una nueva variedad, fragilis, que distinguió por presentar una escultura menos marcada. Mantuvo C. mediterraneus como especie por ser un poco más estrecha y con las áreas algo granuladas.

Bucquoy \& Dautzenberg (1882) admitieron como especie válida a $C$. rissoi e indicaron tres variedades para la especie: var. ex forma 1, meneghinii, definida como: "A sculture plus marqué et stries rayonnantes bien apparents sur les valves antérieure et postérieure, ansi que sur les aires latérales des valves intermédiaires"; var. ex forma 2 , mediterranea, como: "Forme un peu plus allongée que le type et à fossettes bien marquées"; y var. ex forma 3, fragilis descrita como: "De petite taille et à sculpture obsolète, sans aucune trace de stries rayonnantes".

Pilsbry (1892) efectuó un estudio detallado y admitió como especie I. rissoi y las variedades meneghinii y fragilis, diferenciadas por el contraste en la ornamentación; y consideró $C$. mediterraneus como sinónimo de $I$. rissoi, ya que pensaba que la descripción de Reeve (1847) es la misma que para meneghinii.

Ancey (1898) admitió como válida a L. meneghinii, y a su vez propuso la variedad dautzenbergi. Nierstrasz (1906) llevó a cabo un estudio minucioso de I. rissoi, I. meneghinii e I. dautzenbergi, concluyendo que no existe una marcada diferencia entre ellas y verificó la existencia de una amplia gama de ejemplares de transiciones.

Bergenhayn (1931), admitió únicamente a I. rissoi y señaló como sinónimo a $C$. mediterraneus. Sin embargo Thiele (1931), volvió a admitir a Chiton (Lepidopleurus) mediterraneus como una especie diferente de Chiton (Lepidopleurus) rissoi y consideró sinónimo de esta última especie a $C$. meneghinii.

Leloup (1934) realizó un estudio de las estetas de $I$. rissoi, I. meneghinii e $I$. dautzenbergi, observando una misma disposición de éstas para los diferentes ejemplares. Debido a ello corroboró las opiniones de Pilsbry (1892) y Nierstrasz (1906) de admitir como especie a $I$. rissoi y variedades a $I$. meneghinii e I. dautzenbergi. Posteriormente, Leloup \& Volz (1938) mantuvieron como especie a I. rissoi y a $I$. meneghinii como variedad.

A partir de 1962, sólo se ha citado con categoría de especie a I.(I.) rissoi (Malatesta, 1962; Castelló 1973; Kattoulas et al., 1973; Sabelli, 1974; Bosch
\& Sastre, 1976; Barash \& Danin, 1977; Bogi et al., 1980; Leloup, 1980; Panetta et al., 1985; Martín Sintes et al., 1987; Kaas, 1989; Kaas \& Van Belle 1990; Mifsud et al., 1990; Sabelli et al., 1990; Strack, 1990).

Hasta la década de los sesenta, la variación en la ornamentación del tegmento había ocasionado problemas taxonómicos. Según las formas tipos recolectadas, descritas anteriormente, se pueden distinguir las distintas especies y variedades que han propuesto los distintos autores. De tal manera que la Forma 1 se podría corresponder con I. fragilis, la Forma 2 con I. meneghinii, la Forma 3 con I. dautzenbergi y la Forma 4 con I. rissoi y entre esta gama de variación se podrían describir distintas formas diferentes, hecho que corrobora lo apuntado por Nierstrasz (1906).

Aparte de la variabilidad en la ornamentación de I. rissoi descrita por diferentes autores, se ha determinado otra serie de diferencias de los ejemplares estudiados.

Con respecto a la fórmula de líneas de inserción, Pilsbry (1892) indicó para I. rissoi 13/1/10. Nierstrasz (1906), para esta misma especie, 10/1/910 , y para I. meneghinii e I. dautzenbergi $10 / 1 / 9$ y 9-11/1/9-10, respectivamente. Kaas \& Van Belle (1990) anotaron 11-13/1/10-12. En los ejemplares recolectados como $I$. rissoi (Forma 4) esta fórmula presenta un rango de variación de 8-11/1/8-9. El número de líneas para las Formas 1 y 3 es, respectivamente, de 10/1/11 y 10-13/1/9-12.

En relación a las escamas dorsales del perinoto, Nierstrasz (1906) describió un rango de oscilación del número de costillas en las escamas de 9-16 en general para I. rissoi, I. meneghinii e I. dautzenbergi. Kaas \& Van Belle (1990) indicaron 16. En los ejemplares estudiados, el número ha variado en las distintas formas descritas de I. rissoi (Tabla 1).

Kaas \& Van Belle (1990) señalaron que el tamaño de las escamas dorsales es de 120 x $150 \mu \mathrm{m}$. En los ejemplares estudiados se ha encontrado desde $70 \times 30 \mu \mathrm{m}$ hasta $185 \times 65 \mu \mathrm{m}$. Con respecto a las escamas ventrales, Kaas \& Van Belle (1990) indicaron una longitud de $60 \mu \mathrm{m}$ y una anchura de 20 $\mu \mathrm{m}$, sin embargo en los ejemplares estudiados estas dimensiones varían entre 45.0 x $12.5 \mu \mathrm{m}$ y 80 × 20 $\mu \mathrm{m}$. Para las espículas marginales, Kaas \& Van Belle (1990) propusieron un tamaño de 44 x $14 \mu \mathrm{m}$, mientras que el rango de variación en los ejemplares de estudio es de 50 a $65 \mu \mathrm{m}$ de longitud y de 15.0 a $22.5 \mu \mathrm{m}$ de anchura. Todo esto nos lleva a concluir que ejemplares con igual ornamentación del tegmento ofrecen una gran variación de tamaños. 
Nierstrasz (1906), estudió la rádula de I. rissoi, I. meneghinii e I. dautzenbergi sin encontrar diferencias entre ninguna de ellas. En las rádulas observadas de las distintas formas definidas tampoco se han encontrado diferencias y coinciden con la descripción aportada por Kaas \& Van Belle (1990).

Leloup (1934) estudió las estetas sin encontrar diferencias en su disposición para I. rissoi, I. meneghinii e I. dautzenbergi. En el estudio realizado con microscopía electrónica de barrido se ha llegado a la conclusión que la disposición de las estetas es la misma para las diferentes formas.

En general, se puede concluir que $I$. (I.) rissoi presenta una gran variabilidad intraespecífica, en relación a la ornamentación del tegmento, número de líneas de inserción del articulamento, tamaño de las escamas de la cintura y número de costillas de las escamas del perinoto. Mientras que son similares en todas las formas estudidas la rádula y la disposición de las estetas.

\section{Referencias}

ANCEY, C. F., 1898. List of marine shells collected at Port Guedydon, Kabylia, with description of a new Cyclostrema. Nautilus, 12(5): 52-57

Barash, A. \& Danin, Z., 1977. Polyplacophora (Mollusca) from the Eastern Mediterranean. Journal de Conchyologie, 64(1-2): 3-28.

Bergenhayn, J. R. M., 1931. Beitrage zur Malakozoologie der Kanarischen Inseln. Arkiv for zoologi, 13: $1-35$.

Bogi, G., Coppini, M. \& Margelli, A., 1980. Contribution to the knowledge of the molluscan fauna of the central Tyrrhene Sea, Polyplacophora: part 1. Conchiglia, 12(140-141): 14-18.

Bosch, M. \& SASTRE, A., 1976. Sobre la presencia y distribucción de "Polyplacophora" en el litoral de Mallorca. Boletín de la Sociedad de Historia Natural de Baleares, 12: 25-32.

Bucquoy, M. M. E. \& Dautzenberg, P. H., 1882. Les mollusques marins du Rousillon, vol. 1. J.-B. Baillière \& fils. Paris. 509 pp.

CApellini, J., 1859. Catalogue des oscabrions de la Méditerranée, suivi de la description de quelques espèces nouvelles. Journal de Conchyliologie, 7(3): 320-331.

CAstelló, F., 1973. Poliplacóforos de las Islas Baleares. Boletín de la Sociedad de Historia Natural de Baleares, 18: 53-69.

KAAS, P,. 1989. Notes on Loricata. 16. Chitons from the gulf of Gabés, Tunisia. Basteria, 53: 91-92.
KaAs, P. \& Van Belle, R. A., 1990. Monograph of living chitons. 4, Suborder Ischnochitonina: Ischnochitonidae: Ischnochitoninae (continued) Additions to vols 1,2 and 3. E. J. Brill. Leiden. 298 pp.

Kattoulas, M., Koukouras, A. \& Economidis, P., 1973. Benthic fauna of the Evvoia Coast and Evvoia Gulf. Science Annals, Faculty of Physics \& Mathematical, University of Thessaloniki, 13: 17-26.

Leloup, E., 1934. Contribution a la connaissance de la faune des chiton de Villefranche-Sur-Mer et des environs (France Mediterranée). Bulletin de Musée royal d' Histoire naturelle de Belgique, 10(47): 1-20.

Leloup, E., 1980. Chitons de la Mer Rouge, du Golfe de Suez et de la Mediterranée. Bulletin de l'Institute royal de Sciencies naturelle de Belgique, 52(5): 1-17.

Leloup, E. \& Volz, P., 1938. Die Chitonen (Polyplacophoren) der Adria. Thalassia, 2(10): 1-63.

Malatesta, A., 1962. Mediterranean Polyplacophora Cenozoic and Recent. Geologia romana, 1: 145-171.

Martín, D., Dantart, L. \& Ballesteros, M., 1987. Moluscos de las concreciones de algas calcáreas del litoral catalán (NE España). Lavori della Società Italiana di Malacologia, 23: 445-456.

Mifsud, C., Cachia, C. \& Sammut, P. M., 1990. Note sui Poliplacofori delle isole Maltesi. La Conchiglia, 256: 52-61.

Monterosato, T. DE, 1878. Enumerazione e sinonimia delle Conchiglie mediterranee- Monografia dei Chitonidi del Mediterraneo. Giornale di Scienze ed Economiche, Palermo, 14: 9-31.

Nierstrasz, H. F., 1906. Remarks on the chitonidae. Tijdschrift der Nederlandsche Dierkunde Vereeniging, (2), 10: 141-172.

Panetta, P., Dell'Angelo, B. \& Fiordiponti, F., 1985. I Poliplacofori del banco dell' Amendolara (Golfo de Taranto). Oebalia, 11(3): 767-769.

Payraudeau, B. C., 1826. Catalogue descriptif et méthodes des annelides et des mollusques de l'ile de Corse. Paris. 218 pp.

Pilsbry, H. A., 1892-94. Monograph of the Polyplacophora. In: G. W. Tryon. Manual of Conchology. Vol. XIV. Academy of Natural Sciences. Philadelphia. $349 \mathrm{pp}$.

Reeve, L. A., 1847-48. Monograph of the genus Chiton. In: Conchologia iconica: or, Illustrations of the Shells of Molluscous Animals, vol. IV. London. 13 lams.

SABelli, B. A., 1974. Origine e distribuzione dei Poliplacophora viventi in Mediterraneo. Quaderno civico della Stazione idrobiologica di Milano, 5: 7178.

Sabelli. B., Giannnuzzi-Savelli. R. \& Bedulli D., 1990. Catalogo annotato dei Molluschi marini del Mediterraneo, vol. 1. Librería Naturalistica Bolognese. $781 \mathrm{pp}$. 
SHUTtLEWORTH, R. J., 1853. Diagnosen neuer Mollusken. 4, Über den Bau der Chitoniden, mit Aufzhlung der die Antillen und die Canarischen Inseln bewohnenden Arten. Mitteilungen der Naturforschenden Gesellschaft in Bern, 286-291: 169-207.

Strack, H. L., 1990. The distribution of chitons (Polyplacophora) in Greece: addendum. Apex, 5(12): $13-15$
THIELE, J., 1931. Handbuch der systematischen Weichtierkunde, vol. 1. Gustav Fischer. Jena. 34 pp.

Recibido, el 8-VI-1999 Aceptado, el 24-IX-1999 Publicado, el 15-XII-1999 